\title{
Short Communication Prevention of tumour cell dissemination in diagnostic needle procedures
}

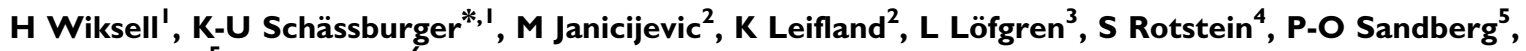 \\ C Wadström ${ }^{5}$ and G Auer
}

'Department of Molecular Medicine and Surgery, Karolinska Institutet, Karolinska University Hospital, Solna (LI:00), 17I76 Stockholm, Sweden; ${ }^{2}$ Department of Mammography, Unilabs AB, Capio S:t Görans Hospital AB, I 281 Stockholm, Sweden; ${ }^{3}$ Department of Surgery, Capio S:t Görans Hospital AB, I I 28 I Stockholm, Sweden; ${ }^{4}$ Department of Oncology-Pathology, Karolinska Institutet, Administration, Building Z I:00, Karolinska University Hospital, 17176 Stockholm, Sweden; ${ }^{5}$ Breast Unit Sabbatsberg's Hospital, Olivecronas väg I, I I 324 Stockholm, Sweden; ${ }^{6}$ Karolinska Biomics Centre, Karolinska Institutet, Karolinska University Hospital, Z5:02, 17176 Stockholm, Sweden

BACKGROUND: A side effect of diagnostic needle biopsies is the possibility to disseminate tumour cells into the needle track, which may cause concern in certain malignant tumour types.

METHODS: In order to prevent tumour cell dissemination we developed a technology that uses radiofrequency (RF) pulses to sterilise the needle track and denaturate tumour cells. To determine feasibility, we applied this technology to fine needle aspiration biopsy (FNAB) and used breast cancer as a model tumour. Routine FNAB was performed in 88 patients with adenocarcinoma and blood droplets passing the skin orifice were cytomorphologically analysed for the presence of tumour cells.

RESULTS: The analysis showed the presence of tumour cells in 65/88 cases (74\%). When using an experimental anti-seeding device in a subset of patients viable tumour cells were found in $0 / 3 \mathrm{I}$ cases $(P<0.00 \mathrm{I})$. In all $3 \mathrm{I}$ patients blood passing the skin orifice was sparse. No degrading effect on the cytological sample inside the needle was detected and pain caused by the RF pulses was comparable to that of the biopsy procedure itself.

CONCLUSION: The herein presented method has the potential to prevent the dissemination of viable tumour cells in the needle track and minimize bleeding without additional pain or degradation of the aspirate.

British Journal of Cancer (2010) I 03, 1706-1709. doi:I0.1038/sj.bjc.6605964 www.bjcancer.com

Published online 2 November 2010

(c) 2010 Cancer Research UK

Keywords: displacement; dissemination; needle biopsy; needle track; seeding; tumour cells

In order to achieve a conclusive diagnosis regarding malignancy in suspicious lesions it is compulsory in the vast majority of situations to perform diagnostic needle biopsies or diagnostic surgery. Concerning diagnostic needle biopsies there are a variety of methods and procedures used which can be subdivided into fine needle aspiration biopsy (FNAB) and core needle biopsy (CNB). Fine needle aspiration biopsy results in samples consisting of single cells and/or minor cell complexes and tissue fragments, whereas CNB results in cut or punched tissue blocks of various volumes. The equipment and methodology to acquire samples are very different for these two methods. The outer diameter of a common FNA needle is in the range of $0.5-0.8 \mathrm{~mm}$, whereas a CNB needle is in the range of $1.2-3 \mathrm{~mm}$ or more. Histological tissue sampled by means of $\mathrm{CNB}$ procedures significantly facilitates morphology-based diagnostic decision making, including the important discrimination between in situ and invasive tumour growth. However, this diagnostic advantage is associated with a

*Correspondence: Dr K-U Schässburger;

E-mail: kai-uwe.schassburger@ki.se

Received 13 August 2010; revised 27 September 2010; accepted 29

September 2010; published online 2 November 2010 higher risk of side effects. Numerous case reports of malignant seeding resulting from needle biopsies have been reported for different tumours (Boutin et al, 1995; Herts and Baker, 1995; Jones et al, 2005; Vaghefi et al, 2005; Al-Leswas et al, 2008) and increasing caution is observed in highly aggressive malignancies, for example in the pancreas (Micames et al, 2003). The true incidence of tumour cell dissemination is hard to determine. This can in part be explained by the fact that it is rather difficult to trace biopsyinduced tumour cell dissemination in surgically removed organs or excised tissue containing the entire or only parts of the needle tracks. Moreover, it may take several years for the disseminated and surviving tumour cells to reach the size of clinically detectable local or peripheral growth. This is further complicated by the fact that the occurrence of needle biopsy-induced local or distant tumour cell growth generally cannot be distinguished from spontaneous tumour recurrence and that post-operative treatment with radiotherapy (Thurfjell et al, 2000) and/or drugs may inhibit spontaneous, as well as biopsy-induced, relapse.

In the present article we report on a technology that is able to denature tumour cells in the needle track after needle biopsies. For this feasibility study we chose breast cancer as a model tumour as it showed favourable characteristics when trying to study local seeding. Blood coming from the skin orifice after final retraction of 
the biopsy needle can be conveniently sampled and analysed for the presence of tumour cells. As FNAB is the standard method of action regarding needle biopsies in breast lesions in Scandinavia, we applied the anti-seeding technology to FNAB procedures.

\section{MATERIALS AND METHODS}

\section{Patients}

This study was approved by the local ethics committee and included 88 patients with adenocarcinomas in the breast diagnosed by FNAB. The first part of the study aimed at establishing breast cancer as a model for tumour cell dissemination. For this purpose routine FNAB procedures were performed in 57 patients at clinical centre 1 . Subsequently 31 patients in clinical centre 2 underwent routine FNAB procedure followed by a second needle biopsy using the anti-seeding device. Patient data are shown in Table 1. All patients were diagnosed according to standard hospital protocols and had a confirmed invasive adenocarcinoma as determined by post-surgical histopathology.

\section{Anti-seeding instrumentation}

The anti-seeding device consists of a control console, as well as a specially designed anti-seeding needle. The needle has an electrically insulated shaft, a length of $50 \mathrm{~mm}$ or $80 \mathrm{~mm}$ and an outer diameter of $0.7 \mathrm{~mm}$. The bare tip, including the bevel section has a length of approximately $6 \mathrm{~mm}$. Energy is delivered in a computer-controlled regime (voltage/current controlled) and triggered by the operator's needle movements by means of a miniaturised accelerometer. The applied symmetrical burst pulses have a $55 \mathrm{~ms}$ linear ramp, a duration of $200 \mathrm{~ms}$ and a radiofrequency (RF) period of $3.3 \mu$ s. Every burst pulse incorporates around 60000 periods and delivers an energy of approximately $6 \mathrm{~J}$. Consecutive burst pulses are separated by a minimum time interval. Additionally, pulses are blocked if the velocity of the needle is too low in order to ensure sufficient heat conduction. The tissue cell sample, which is protected by the Faraday's cage realized by the needle internal channel cavity, is thus fully protected from heat degradation. Skin burn is avoided by blocking energy pulses in proximity to the skin orifice. The entire prototype, including the needle, was designed by NeoDynamics, Sweden, under the lead of HW The device is conform to the international safety norm EN 60601-1. It meets the insulation demands of cardiac floating regarding patient applied parts, that is, a frequency weighted patient leakage current of less or equal to $10 \mu \mathrm{A}$, which is lower than that required to disturb the cardiac activity even in direct contact.

Table I Clinical and histopathological data on patients included in the study

\begin{tabular}{lcccc}
\hline & \multicolumn{2}{c}{ Clinical Centre I } & \multicolumn{2}{c}{ Clinical Centre 2 } \\
\hline No. of patients & 57 & & 31 & \\
Tumour size (mm) & $23.7 \pm 13.1$ (s.d.) & \multicolumn{1}{c}{$17.0 \pm 7.8$ (s.d.) } \\
Histological type & No. & $\%$ & No. & $\%$ \\
$\quad$ Ductal & 43 & 75 & 26 & 84 \\
Lobular & 12 & 21 & 4 & 13 \\
Others & 2 & 4 & 1 & 3 \\
Malignancy grade (Elston) & No & $\%$ & No & $\%$ \\
I & 2 & 4 & 6 & 19 \\
II & 35 & 61 & 21 & 68 \\
III & 20 & 35 & 4 & 13 \\
\hline
\end{tabular}

\section{Biopsy protocol}

In 57 patients at the clinical centre 1 diagnostic FNAB procedures were performed by two experienced cytologists (GA and P-OS) using needles with an outer diameter of $0.7 \mathrm{~mm}$ (Sterican, B. Braun AG). Fine needle aspiration biopsy was performed without local anaesthesia and without ultrasound guidance. The needle was mounted on a standard syringe hand-piece (Cameco, Medical Lth., Great Britain, UK). After entering the tumour the needle passed the mass 10-15 times in various directions in order to collect representative material from the entire tumour mass. In 31 patients at clinical centre 2 ultrasound-guided FNAB was performed by an experienced radiologist (MJ). The lesions were detected using an US instrument (Philips iU22). While performing the biopsy, the radiologist held the needle at the base while it was connected with flexible tubing to the syringe, manoeuvred by a technician. Around 10 to 15 passes through the lesion were performed in order to acquire a sufficiently high sampling yield. In these 31 patients a subsequent biopsy procedure was performed incorporating the anti-seeding device. The needle was inserted through the skin at a different location than the previous FNAB procedure. While the needle was passed through the lesion back and forth, as well as finally retracted, burst pulses were applied. Immediately after the biopsy procedure blood/secretion droplets emerging from the skin orifice were sampled on a glass slide. The samples were air-dried, May-Grünwald-Giemsa stained and microscopically analysed by two experienced cytologists (GA and P-OS).

\section{RESULTS}

The results following FNAB procedures with and without the antiseeding device are shown in Table 2. Seeding frequencies in standard FNAB procedures at the two clinical centres did not differ significantly (Fisher's exact test, two-sided, $P=0.4466$ ). In total, tumour cells in the blood droplet/s were present in 65 of the 88 cases analysed at the two centres (74\%). Breast cancer showed to be an adequate model tumour for studying local seeding. Blood passing the skin orifice could easily be sampled and analysed. Protocols from clinical centre 1 were adopted for the study at centre 2 . Fine needle aspiration biopsy procedures using the antiseeding needle were performed in 31 cases at clinical centre 2 without complications. Induced pain was comparable to that of routine fine needle aspiration procedures. The acquired aspirate showed no sign of degradation. As shown in Figure 1, the RF burst pulses could be detected on the US image. Bleeding was significantly reduced as compared with common FNAB. By applying light mechanical pressure to the breast, secretion was extracted from the needle track. Tumour cells in the secretion were found in only 1 of 31 cases (3\%) after anti-seeding needle performance, (McNemar's test, two sided, $P<0.001$ ). None of the tumour cells in the solitary positive sample after the anti-seeding needle biopsy were judged to be morphologically well preserved.

Table 2 Presence of tumour cells in sampled blood droplets/secretion passing the skin orifice after FNAB with and without anti-seeding device

\begin{tabular}{lcc}
\hline & No & \% Positive \\
\hline Clinical centre 1 & 57 & 77 \\
FNAB & & \\
Clinical centre 2 & 31 & 68 \\
FNAB & 31 & $3^{\text {a }}$ \\
FNAB with anti-seeding & & \\
\hline
\end{tabular}

Abbreviation: $\mathrm{FNAB}=$ fine needle aspiration biopsy. ${ }^{\mathrm{a}} \mathrm{Cytomorphologically} \mathrm{degener-}$ ated tumour cells (see Figure 4) 


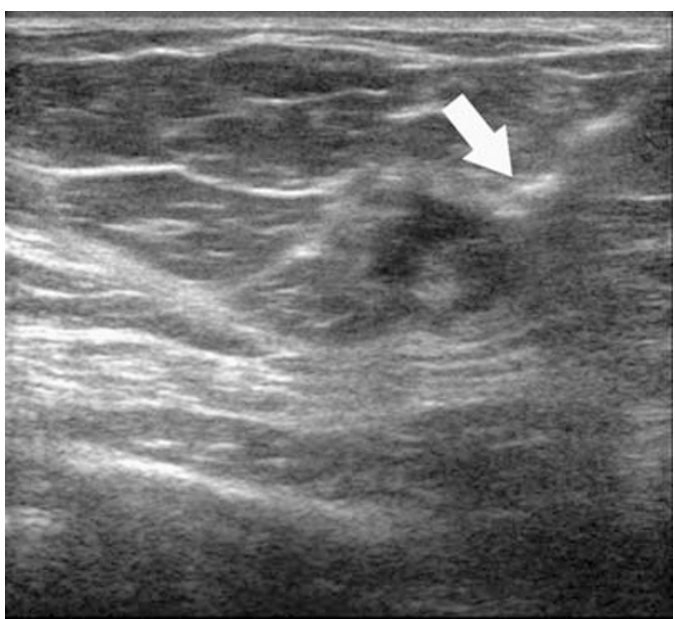

Figure I US image taken during FNAB with anti-seeding. The tumour mass can be seen in the centre with the needle entering from top right. The anti-seeding pulse shows on the image as a short flash (arrow).

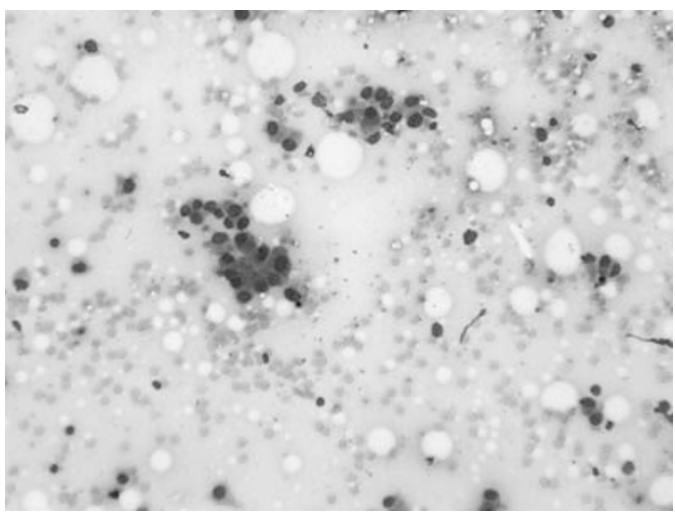

Figure 2 May-Grünwald-Giemsa-stained fine needle aspirate from a breast adenocarcinoma using the anti-seeding technique. The cellular material is well preserved and can be analysed according to generally used cytological criteria (magnification $\times 20$ ).

\section{Cytological analysis}

Cytological analysis of the aspirate after FNAB with anti-seeding showed that the sample was not distinguishable from an aspirate acquired by standard FNAB (Figure 2). Analysis of the blood droplet/s after common FNAB showed different levels of tumour cell concentrations. The aim of this study, however, was not to quantify tumour cell concentration. Figure 3 shows a smear of a sampled blood droplet after standard FNAB. The cytological analysis of the secretion after FNAB with anti-seeding showed a significantly reduced number of erythrocytes and one case presenting tumour cells that were judged cytomorphologically degenerated (Figure 4).

\section{DISCUSSION}

Today the battle against malignant diseases is focused on early diagnosis and treatment. When a malignant disease is diagnosed, the next step is to determine histopathological characterisation and malignancy potential, clinical stage, as well as an increasing number of biological tumour markers. On the basis of the results of these investigations a decision is made concerning treatment

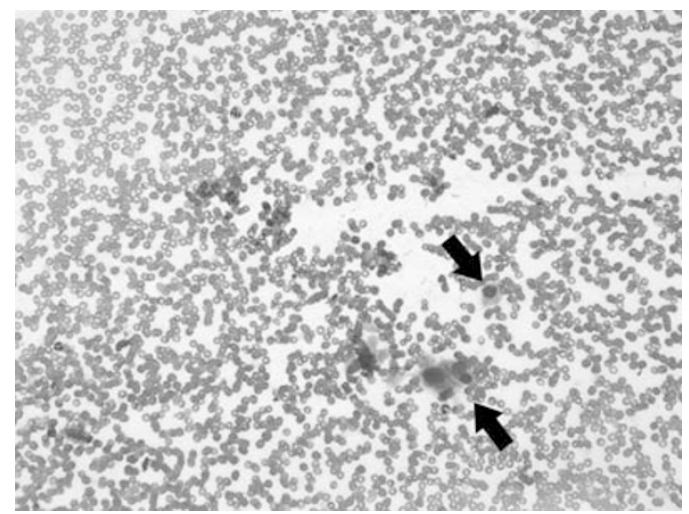

Figure 3 May-Grünwald-Giemsa-stained smear of a blood droplet that passed the skin orifice after retraction of the FNA needle from a breast adenocarcinoma without using the anti-seeding technique. Well-preserved cancer cells (arrows) can be seen surrounded by peripheral blood cells (magnification $\times 20)$.

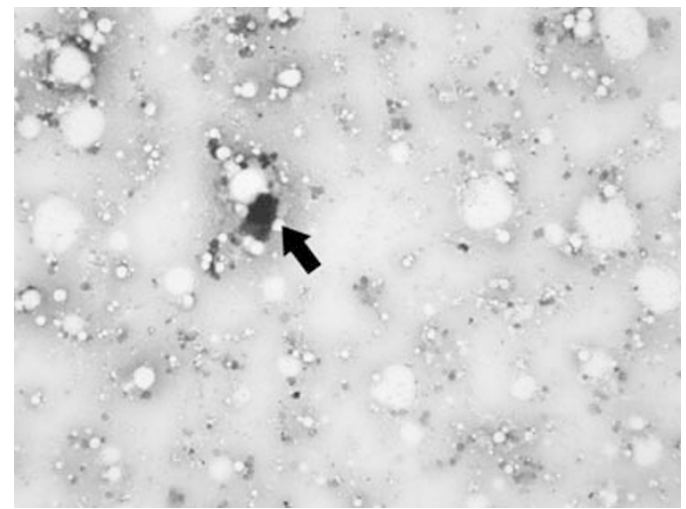

Figure 4 May-Grünwald-Giemsa-stained smear of a droplet of fluid that passed the skin orifice after retraction of the FNA needle from a breast adenocarcinoma using the anti-seeding technique. Only a minor number of blood cells and denatured cancer cells (arrow) can be seen (magnification $\times 20)$.

that can be of local and/or general mode, depending on morphological characteristics, the spread of disease and biological markers. In order to obtain a conclusive diagnosis it is generally accepted to use invasive cell- or tissue-sampling methods, that is, FNAB, CNB or surgical biopsy. Dissemination of tumour cells during biopsies is a recognised complication of these procedures and a cause of concern in certain aggressive tumour forms (Micames et al, 2003; Rodgers et al, 2003). However, its true incidence and clinical significance is hard to determine. In the literature, prophylactic measures against needle track seeding include surgical resection (Intra et al, 2005), radiotherapy (Espinoza et al, 2005) and cauterisation of the needle tract (Liang et al, 2009). However, surgery and radiotherapy are rarely feasible being both costly and time intensive. Cauterisation of the needle track has been reported for treatment electrodes used in therapeutic modalities applying $\mathrm{RF}$ and microwave ablation, in which the treatment electrode is being retracted while still active (Murakami et al, 1995; Buscarini and Buscarini, 2004; Wiksell et al, 2010).

We herein present a lean and patient-friendly technology that diminishes the risk of tumour cell seeding when performing needle biopsy procedures. To our knowledge this new technique for the first time combines diagnosis with local minimal treatment 
preventing tumour cell dissemination by means of RF burst pulses. The aspirate inside the needle is not degraded because of the sample being protected within the Faraday's cage realized by the needle cavity. Furthermore the movement of the needle provides forced cooling to the sample inside, following needle exposure to dissipatively heated tissues. The anti-seeding procedure was safe and resulted in no complications for the patient. Tumour cells that were disseminated into the needle track were denatured and bleeding was significantly reduced. Using the breast tumour as a model in this feasibility study proved to be suitable. For standard fine needle procedures, the blood droplet/s passing the skin orifice were easily sampled and analysed. As the anti-seeding procedure reduced the amount of blood in the needle channel, bleeding was reduced and a light mechanical pressure had to be applied in order to obtain fluid from inside the needle channel. As no wellpreserved tumour cells were present in the secretion that passed the skin orifice, it is reasonable to assume that they were denatured in the needle track. This is further underlined by the denatured tumour cells found in one solitary case. The reason that needle biopsy procedures with anti-seeding were performed after common FNAB is because of the ethical considerations that aimed at securing a FNAB sample before applying the new technology.

\section{REFERENCES}

Al-Leswas D, O'Reilly DA, Poston GJ (2008) Biopsy of solid liver tumors: adverse consequences. Hepatobiliary Pancreat Dis Int 7: 325-327

Boutin C, Rey F, Viallat JR (1995) Prevention of malignant seeding after invasive diagnostic procedures in patients with pleural mesothelioma. A randomized trial of local radiotherapy. Chest 108: $754-758$

Buscarini E, Buscarini L (2004) Radiofrequency thermal ablation with expandable needle of focal liver malignancies: complication report. Eur Radiol 14: 31 - 37

Espinoza S, Briggs P, Duret JS, Lapeyre M, de Baere T (2005) Radiofrequency ablation of needle tract seeding in hepatocellular carcinoma. J Vasc Interv Radiol 16: $743-746$

Herts BR, Baker ME (1995) The current role of percutaneous biopsy in the evaluation of renal masses. Semin Urol Oncol 13: 254-261

Intra M, Mazzarol G, Rietjens M, Diaz Brito JA, Gennari R, Soteldo J, Rodriguez J, Bassani G, Bassi F (2005) Extramammary recurrence of DCIS after total mastectomy: an iatrogenic displacement following needling procedures? Breast J 11: $297-300$

Jones OM, Rees M, John TG, Bygrave S, Plant G (2005) Biopsy of resectable colorectal liver metastases causes tumour dissemination and adversely affects survival after liver resection. Br J Surg 92: 1165-1168

Liang P, Wang Y, Yu X, Dong B (2009) Malignant liver tumors: treatment with percutaneous microwave ablation - complications among cohort of 1136 patients. Radiology 251: $933-940$

Micames C, Jowell PS, White R, Paulson E, Nelson R, Morse M, Hurwitz H, Pappas T, Tyler D, McGrath K (2003) Lower frequency of peritoneal carcinomatosis in patients with pancreatic cancer diagnosed
However, as different needle biopsy locations were used this does not constitute a weakness of the study that aimed at establishing feasibility. Radiofrequency pulses applied inside the tumour and the associated reduction of bleeding could possibly have effects on the dissemination of tumour cells into blood and lymphatic vessels. The presented technology has the potential to denature cancer cells in the needle track without affecting the aspiration sample or causing additional pain to the patient. The data obtained herein motivates a randomized clinical trial with long-term followup comprising highly aggressive malignancies in which tumour spread is a well-known major complication. The technology can hopefully be applied to other needle biopsy modalities, such as the widely used CNB and contribute to improve the safety of needlebased diagnostics.

\section{ACKNOWLEDGEMENTS}

This study was supported by the 7th Framework Programme of the European Union (FLUODIAMON), VINNOVA and AFA Insurance, Sweden. Special thanks to Louise Rosenberg for her help with handling the patient journals. by EUS-guided FNA vs percutaneous FNA. Gastrointest Endosc 58: $690-695$

Murakami R, Yoshimatsu S, Yamashita Y, Matsukawa T, Takahashi M, Sagara K (1995) Treatment of hepatocellular carcinoma: value of percutaneous microwave coagulation. AJR Am J Roentgenol 164: 1159-1164

Rodgers MS, Collinson R, Desai S, Stubbs RS, McCall JL (2003) Risk of dissemination with biopsy of colorectal liver metastases. Dis Colon Rectum 46: 454-458; discussion 8-9

Thurfjell MG, Jansson T, Nordgren H, Bergh J, Lindgren A, Thurfjell E (2000) Local breast cancer recurrence caused by mammographically guided punctures. Acta Radiol 41: 435-440

Vaghefi H, Magi-Galluzzi C, Klein EA (2005) Local recurrence of prostate cancer in rectal submucosa after transrectal needle biopsy and radical prostatectomy. Urology 66: 881

Wiksell H, Löfgren L, Schässburger KU, Grundström H, Janicijevic M, Lagerstedt U, Leifland K, Nybom R, Rotstein S, Saracco A, Schultz I, Thorneman K, Wadström C, Westman L, Wigzell H, Wilczek B, Auer G, Sandstedt B (2010) Feasibility study on the treatment of small breast carcinoma using percutaneous US-guided preferential radiofrequency ablation (PRFA). Breast 19: 219-225

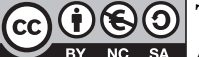

This work is licensed under the Creative Commons Attribution-NonCommercial-Share Alike 3.0 Unported License. To view a copy of this license, visit http://creativecommons. org/licenses/by-nc-sa/3.0/ 